\title{
Fertility and Cancer-Patient, Physician, and Institutional Barriers
}

\author{
Gwendolyn P Quinn, PhD ${ }^{1,2}$ and Susan T Vadaparampil, PhD, MPH ${ }^{1,2}$ \\ 1. Associate Member, Moffitt Cancer Center and Research Institute; \\ 2. Associate Professor, Department of Oncologic Science, University of South Florida, College of Medicine
}

\begin{abstract}
Loss of fertility is often a negative consequence of cancer treatments that include chemotherapy and radiation. Cancer survivors who experience infertility are at increased risk for emotional distress and reduced quality of life. Fertility preservation options are available for cancer patients of childbearing age. Many cancer patients are greatly concerned about the loss of fertility and are interested in fertility preservation. Most preservation options are available only prior to the initiation of cancer treatment. Communication within this limited time-frame concerning fertility loss and fertility preservation options for newly diagnosed cancer patients is imperative. There are considerable barriers at the patient, physician, and system level that impede discussion and pursuit of fertility preservation for the newly diagnosed cancer patient. Barriers to fertility preservation include lack of physician referral, need for patient education resources, and financial costs.
\end{abstract}

\section{Keywords}

Infertility, oncology, patient-provider communication, fertility preservation, survivorship

Disclosure: The authors have no conflicts of interest to declare.

Received: August 12, 2009 Accepted: October 6, 2010 Citation: US Oncology \& Hematology, 2011;7(1):22-4 DOl: 10.17925/OHR.2011.07.1.22

Correspondence: Gwendolyn Quinn, PhD, Moffitt Cancer Center, 12902 Magnolia Drive, MRC CANCONT, Tampa, FL 33612. E: gwen.quinn@moffitt.org

There are currently over 450,000 cancer survivors between 19 and 39 years of age. In 2005, ${ }^{1-3} 1,372,910$ people were diagnosed with cancer and $4 \%$ ( 55,000 cases) were under 35 years of age. ${ }^{2,3}$ Breast cancer, cervical cancer, non-Hodgkin's Iymphoma, leukemia, and melanoma are the most common incident cancers in people under 40 years of age. ${ }^{3}$ Infertility is an expected consequence of most cancer treatments that include chemotherapy and/or radiotherapy. ${ }^{4}$ While the exact rate of infertility by cancer site, treatment modality, or in general is largely unknown, ${ }^{4-6}$ it is estimated that sustained infertility develops in 50-95\% of cancer survivors, especially when bone marrow transplants or combination therapies are used. ${ }^{4,6,7}$

There are options for preserving the fertility of some cancer patients and the American Society of Clinical Oncology (ASCO) recommends these options be discussed-and in most cases pursued-prior to the initiation of treatment. Fertility, childbearing, and parenting are significantly related to quality of life for cancer survivors. Loss of fertility is one negative consequence of cancer treatment that can adversely affect quality of life $e^{2,5,8-10}$ for survivors. There are considerable barriers at the patient, physician, and system level, however, that impede discussion and pursuit of fertility preservation for the newly diagnosed cancer patient.

\section{Fertility and Quality of Life}

Many cancer patients are interested in fertility preservation and prefer to have biological children rather than pursuing adoption or third-party reproduction. ${ }^{5,10-12}$ Cancer survivors who are free of disease often believe their diagnosis will make them better parents. ${ }^{10}$ In addition, adoption agencies may reject cancer survivors based on their health histories, even with recommendations from physicians reporting the survivor is cancer-free. ${ }^{13}$ Studies of cancer patients report that loss of fertility is an immense concern that may cause great distress. , $, 7-9,11,14-16$ This mirrors findings in studies of infertile non-cancer populations as well those cancer survivors whose quality of life is often affected by discontent from the grief of infertility. ${ }^{12,17}$

\section{Fertility Preservation Options}

Male cancer patients who undergo chemotherapy may experience toxicity to the gonads that may lead to permanent spermatogenesis impairment. ${ }^{18,19}$ In general, men have had a longer history of availability of fertility preservation options, particularly semen cryopreservation. With the recent advances in cryopreservation of testicular tissue and semen, samples can be taken from most post-pubertal males. ASCO and the American Society of Reproductive Medicine (ASRM) recommend that all male cancer patients be offered the option of semen cryopreservation and referred to a licensed facility prior to the initiation of cancer treatment. . $^{720}$

For women, fertility and cancer encompass more complex issues. ${ }^{21}$ Ovarian transposition or embryo cryopreservation are the established options available to women. ${ }^{4,7}$ Ovarian transposition is a surgical technique used to protect ovarian function before the delivery of gonadocidal doses of radiation therapy by moving the ovaries out of the field of radiation. Ovarian transposition is typically performed in 
patients whose treatment includes pelvic radiotherapy as a part of the management of Hodgkin's disease and other gynecological malignancies.

Embryo cryopreservation requires controlled ovarian hyperstimulation and oocyte retrieval with cryopreservation of embryos prior to, or during, an appropriate break in cancer treatment. ${ }^{8}$ This type of preservation is unsuitable for patients without a male partner, however, or who for those decline donor sperm. ${ }^{4}$

Additional preservation options exist that are considered experimental. Ovarian cryopreservation, where the egg-containing ovarian cortex is sliced thinly and the tissue slices are cryopreserved and frozen using equipment designed to produce a slow and controlled freezing process, is the most recent advance in fertility preservation. When clinical circumstances are favorable, retransplantation of the ovarian tissue strips can be attempted. Low-temperature banking of ovarian tissue can be done with pre- and post-pubertal female cancer patients. Oocyte cryopreservation involves the extraction of eggs that are frozen and stored. The eggs are then thawed, fertilized, and transferred to the uterus as embryos at such time when the female patient is physically able to attempt pregnancy.

The procedures associated with embryo and oocyte cryopreservation can take several weeks to be performed, which may lead to a delay in cancer therapy-a less appealing option for those diagnosed with advanced-stage cancers. ${ }^{4,7,10}$

ASCO provides a list of fertility preservation options for men and women accompanied by eligibility criteria and considerations. ${ }^{4}$

\section{Patient-Provider Communication About Fertility Preservation}

Despite evidence that patients want information about future fertility, the existing research finds large gaps between recommended and actual clinical practice related to the discussion of options with cancer patients. A pilot study, ${ }^{10}$ as well as a follow-up study, ${ }^{22}$ revealed that patients are not well informed about available preservation options. Cancer survivors were queried about whether a healthcare provider had discussed the risk for infertility with them. Only 50\% of survivors recalled receiving information from a healthcare provider about the risks of infertility from their treatment. ${ }^{10,22}$ Furthermore, $<35 \%$ of the women in a study of breast cancer survivors recalled discussing the risks of pregnancy during or after cancer treatment with a healthcare provider. ${ }^{23}$

In the past few years two leading professional organizations have issued guidelines specific to fertility preservation in cancer patients. In 2005, ASRM published guidelines specifically developed for oncologists about the discussion of options for young cancer patients. ${ }^{20}$ In 2006, ASCO published guidelines stating that "oncologists should address the possibility of infertility with patients treated during their reproductive years and be prepared to discuss possible fertility preservation options or refer appropriate and interested patients to reproductive specialists. Clinician judgment should be employed in the timing of raising this issue, but discussion at the earliest possible opportunity is encouraged." Until this time, there had been no official practice guidelines for such discussions, which may have contributed to the current lack of discussion about fertility preservation between patients and providers. Despite lack of patient recall about discussion of infertility, by contrast, a recent national survey of US oncologists showed that $74 \%$ said they always discuss fertility preservation with their patients of childbearing age. ${ }^{25}$

Several factors may contribute to lack of discussion by oncologists, including the physician's knowledge and attitudes toward fertility preservation or comfort with the topic. ${ }^{24-26}$ Physicians may feel that treating the cancer is the highest priority for cancer care. ${ }^{24-27}$ A physician may bring up the topic of fertility, but patients can be overwhelmed by their diagnosis and only want to focus on surviving the disease or not recall that the topic was mentioned. ${ }^{24-26}$ Physicians may not want to press the issue of preservation with patients whose sole focus at the time of diagnosis is survival. ${ }^{24,26}$

Physicians may also be reluctant to have this discussion with patients who have a poor prognosis for survival. ${ }^{24-27}$ The issue of a physician's personal/ethical concerns with posthumous parenting have been identified in previous studies as a deterrent to discussing fertility. ${ }^{26,27}$ Quinn et al. in a 2009 survey of US oncologists found that only 33\% agreed with the statement 'I support posthumous parenting (child born from assisted reproduction subsequent to the patient's death).' Although some physicians disagree with posthumous parenting, ASRM recommends that physicians do not deny patients fertility preservation information based on their personal beliefs. ${ }^{20}$

\section{Referrals for Fertility Preservation}

$\mathrm{ASCO}^{4}$ and $\mathrm{ASRM}^{20}$ guidelines, as well as the American Cancer Society, Fertile Hope Organization, Lance Armstrong Foundation, and other organizations, recommend that newly diagnosed cancer patients receive a referral to a reproductive endocrinology infertility specialist (REIS). REIS and associated clinics offer counseling and fertility preservation services tailored to the individual risk for the patient based on factors such as age, cancer type and stage, and the therapeutic agent recommended for treatment.

Little is known about the most effective ways to refer patients to REIS. Although differences exist in the treatment planning for each cancer, there are opportunities to refer and educate patients about fertility preservation during the treatment planning process. In 2009 Quinn et al. conducted a national survey of US oncologists and found that $47 \%$ of respondents routinely referred cancer patients of childbearing age to a reproductive endocrinologist. ${ }^{25}$ The most commonly identified barrier to referrals was the physician's perceptions of the patient's inability to delay treatment (35\%), rather than a lack of resources or known referral sources (9\%). ${ }^{25}$

\section{System Barriers}

\section{Fertility Preservation Resources/Materials}

According to the Fertile Hope Organization, two-thirds of physicians would be more likely to discuss fertility preservation if detailed patient education materials were available. ${ }^{28}$ Advocacy organizations, such as Fertile Hope, the Lance Armstrong Foundation, and the American Cancer Society, have been proactive in making this information about fertility preservation available to cancer patients and their families. For example, Fertile Hope provides information on the impact of cancer on fertility, possible options, and resources related to preservation. ${ }^{29}$ The Lance 
Armstrong Foundation has similar resources tailored to the needs of male and female patients. ${ }^{30,31}$ Despite these readily available patient education resources, a 2009 survey of US oncologists showed that fewer than $35 \%$ routinely provide patients with any fertility preservation educational materials. ${ }^{25}$

The physician's perception of a patient's insurance status, availability of cryopreservation resources, and cost of procedures may also serve as barriers. ${ }^{24,26}$ Several single-institution studies have identified the costs associated with fertility preservation as a barrier to the discussion. Schover's 2002 study at a Texas institution and the Quinn et al. 2007 study of an institution in Florida found the financial costs associated with fertility preservation served as barriers to the discussion. ${ }^{24,27}$ In a qualitative study of pediatric oncologists in the state of Florida, physicians perceived the costs of preservation and lack of insurance coverage as a primary barrier for the majority of patients. ${ }^{26}$

\section{Financial Costs Associated with Fertility Preservation}

The average national cost of sperm banking is approximately $\$ 350-600 / y e a r$. Embryo cryopreservation may cost up to $\$ 500$ for the necessary ovarian stimulation drugs, between $\$ 5,000$ and $\$ 10,000$ for egg harvesting, and an additional $\$ 10,000$ for each attempt at embryo transfer. ${ }^{28}$ To date, the majority of insurance companies do not cover fertility preservation for the newly diagnosed cancer patient. ${ }^{32}$

Quinn et al. in 2009 examined codified state policies related to infertility and assessed their relevance for cancer patients. ${ }^{32}$ In fact, the majority of cancer patients do not meet the legal definition of 'infertile' as defined by most state laws. Fifteen states (29.4\%) had laws relating to insurance coverage for infertility or in vitro fertilization (IVF) procedures and two states (California and Illinois) specifically excluded IVF from a mandatory offer of coverage. The majority of states define infertility as the inability to conceive a child after one year of unprotected sexual intercourse. This definition was developed for the general infertile population and is not responsive to the unique circumstances of cancer patients or survivors. ${ }^{20,32}$

\section{Conclusion}

Infertility may be a side effect of cancer treatment for many cancer survivors. Despite both established and experimental options available for cancer survivors prior to the start of cancer treatment, discussion of fertility preservation options, use of educational materials, and referrals to REIS by healthcare providers remains low. Moderate to low rates of discussion and referral can be attributed to patient factors, such as the inability of the patient to delay treatment or the patient's resistance to addressing the issue at the time of diagnosis. Physician factors can also play a role, as in the case where the physician elects not to discuss the topic due to a patient's poor prognosis or a personal discomfort with ethical issues, such as posthumous parenting. Finally, system factors may also serve as obstacles, with lack of distribution of education materials, financial constraints, and third-party payor limitations.

Cancer survivors who experience infertility are at increased risk for emotional distress and reduced quality of life. ${ }^{10}$ By providing survivors with timely relevant information related to their fertility preservation options, it may be possible to reduce emotional distress and improve quality of life as they transition from cancer patient to survivor.
1. Jemal A, Siegel $\mathrm{R}$, Ward $\mathrm{E}$, et al., Cancer statistics, 2007, CA Cancer I Clin, 2007;57:43-66.

2. American Cancer Society. Cancer Facts and Figures 2005. Atlanta: American Cancer Society; 2005. Available at: www.cancer.org /acs/groups/content/@nho/documents/document/caff2005f4pwse curedpdf.pdf (accessed February 16, 2011).

3. American Cancer Society, Cancer Facts and Figures 2008, ACS, 2008 Available at: www.209.135.47.118/downloads/STT/

2008CAFFfinalsecured.pdf (accessed February 16, 2011)

4. Lee SJ, Schover LR, Partridge AH, et al., American society of clinical oncology recommendations on fertility preservation in cancer patients, I Clin Oncol, 2006;24:2917-31.

5. Zebrack BJ, Casillas J, Nohr L, et al., Fertility issues for young adult survivors of childhood cancer, Psychooncology, 2004:13:689-99.

6. Oktay K, Cil AP, Bang H, Efficiency of oocyte cryopreservation: a meta-analysis, Fertil Steril, 2006;86:70-80

7. Wallace $W H$, Anderson RA, Irvine DS, Fertility preservation for young patients with cancer: who is at risk and what can be offered? Lancet Oncol, 2005;6:209-18.

8. Sonmezer $\mathrm{M}$, Oktay $\mathrm{K}$, Fertility preservation in female patients, Hum Reprod Update, 2004;10:251-66.

9. Deimling GT, Kahana B, Bowman KF, et al., Cancer survivorship and psychological distress in later life Psychooncology, 2002;11:479-94.

10. Schover LR, Rybicki LA, Martin BA, et al., Having children afte cancer. A pilot survey of survivors' attitudes and experiences, Cancer, 1999:86:697-709.

11. Wenzel L, Donnelly J, Fowler J, et al., Resilience, reflection, and residual stress in ovarian cancer survivorship: a gynecologic oncology group study, Psychooncology, 2002;11:142-53.
12. Canada $A L$, Schover $L R$, Research promoting better patient education on reproductive health after cancer, J Natl Cancer Inst Monogr, 2005;34:98-100.

13. Rosen A, Third-party reproduction and adoption in cance patients, I Natl Cancer Inst Monogr, 2005:91-3.

14. Cope D, Patients' and physicians' experiences with sperm banking and infertility issues related to cancer treatment, Clin I Oncol Nurs, 2002:6:293-4.

15. Bodurka-Bevers $D$, Basen-Engquist $K$, Carmack $C L$, et al., Depression, anxiety, and quality of life in patients with epithelial ovarian cancer, Gynecol Oncol, 2000;78:302-8.

16. Gotay CC, Muraoka MY, Quality of life in long-term survivors of adult-onset cancers, J Natl Cancer Inst, 1998;90:656-67.

17. King BR, Subfecundity and anxiety in a nationally representative sample, Soc Sci Med, 2003:56:739-51.

18. King L, Quinn GP, Vadaparampil ST, et al., Oncology nurses' perceptions of barriers to discussion of fertility preservation with patients with cancer, Clin I Oncol Nurs, 2008;12:467-76.

19. Glaser A, Wilkey O, Greenberg M, Sperm and ova conservation: existing standards of practice in North America, Med Pediatr Oncol, 2000;35:114-8.

20. Ethics Committee of the American Society for Reproductive Medicine, Fertility preservation and reproduction in cancer patients, Fertil Steril, 2005;83:1622-8.

21. Quinn GP, Vadaparampil ST, Bell-Ellison BA, et al., Patientphysician communication barriers regarding fertility preservation among newly diagnosed cancer patients, soc Sci Med, 2008;66:784-9.

22. Schover $L R$, Brey K, Lichtin $A$, et al., Knowledge and experience regarding cancer, infertility, and sperm banking in younger male survivors, J Clin Oncol, 2002;20:1880-9.
23. Duffy CM, Allen SM, Clark MA, Discussions regarding reproductive health in young women with breast cancer undergoing chemotherapy, I Clin Oncol, 2005;23:766-73.

24. Quinn G, Vadaparampil S, Gwede C, et al., Discussion of fertility preservation with newly diagnosed patients: oncologists' views, I Cancer Surviv, 2007;1:146-55.

25. Quinn $\mathrm{G}$, Vadaparampil $\mathrm{S}$, Lee $\mathrm{JH}$, et al., Physician referral for fertility preservation with oncology patients: a national study of practice behaviors, I Clin Oncol, 2009;27:5952-7.

26. Vadaparampil S, Quinn $\mathrm{G}$, King L, et al., Barriers to fertility preservation among florida pediatric oncologists, Patient Educ Couns, 2008:72:402-10.

27. Schover LR, Brey K, Lichtin A, et al., Oncologists' attitudes and practices regarding banking sperm before cancer treatment, Clin Oncol, 2002;20:1890-7.

28. FertileHope, Parenthood Options, FertileHope, 2006. Available at: www.fertilehope.org/learn-more/cancer-and-fertilityinfo/parenthood-options.cfm (accessed February 16, 2011)

29. FertileHope, Statistics, FertileHope, 2006. Available at: www.fertilehope.org/learn-more/index.cfm (accessed February 16, 2011).

30. Adler NE, Page A, eds, Institute of Medicine, Committee on Psychosocial Services to Cancer Patients/Families in a Community S, Cancer Care for the Whole Patient: Meeting Psychosocial Health Needs, Washington DC, National Academies Press, 2008.

31. National Cancer Institute, The Nation's Investment in Cancer Research: Connecting the Cancer Community, 2008. Available at: plan.cancer.gov/pdf/nci_2009_plan.pdf

32. Vadaparampil ST, Lowrey KM, Miree CA, et al., State laws and regulations covering fertility preservation for cancer patients, Clin Oncol, 2009;27(15S): abstract 9509. 